\title{
Detection of surface plasmons by scanning tunneling microscopy
}

\author{
R. Möller, U. Albrecht, J. Boneberg, B. Koslowski, P. Leiderer, and K. Dransfeld \\ Fakultät für Physik der Universität Konstanz, D-7750 Konstanz, Germany
}

(Received 24 July 1990; accepted 4 December 1990)

The influence of surface plasmons excited in a polycrystalline silver film on the tunneling current of a scanning tunneling microscope (STM) has been analyzed. The plasmons cause an additional flow of electrons from the tungsten tip to the silver surface on the order of up to $50 \mathrm{pA}$. This process is independent of the polarity of the applied bias voltage, thereby excluding effects of thermal expansion. The different nature of the ordinary tunneling current and the surface plasmon induced current is clearly revealed by their different dependence on the gap distance. The local distribution of the intensity of the surface plasmon induced signal reveals structures on a nanometer scale. Some of them are correlated to the surface topography.

\section{INTRODUCTION}

The interaction of visible light with electrons tunneling through a vacuum gap is of fundamental interest. Different mechanisms for this interaction have been proposed and some of them have been verified experimentally. Amer et al. reported the thermal expansion of the tunneling tip and possibly the sample surface upon radiation with visible laser light. The use of the nonlinearity of the current voitage characteristic for the direct rectification of light has been discussed by Cutler $e t$ al. ${ }^{2}$ They proposed to determine the traversal time for tunneling of the electrons by the cut-off frequency above which the rectification process would no longer operate. Mixing of two laser frequencies in the tunneling gap has been demonstrated by Krieger et al. ${ }^{3}$ and Arnold et $a l .^{+}$for frequency differences ranging from $\mathrm{MHz}$ up to several THz. These results have been explained by thermal expansion of the outermost part of the tip at low frequencies and by the nonlinearity of the current-voltage characteristic at high frequencies. On semiconducting surfaces a photovoltage in the order of a few $100 \mathrm{~mW}$ has been observed upon radiation with laser light. ${ }^{5,6}$

Gimzewski et al. ${ }^{7}$ have observed the emission of light from a tunneling junction when tunneling on silver surfaces. This effect has been attributed to the excitation of surface plasmons by inelastic tunneling of electrons. ${ }^{8}$ Here the observed photons resilt from the decay of the plasmons. Recently the detection of laminescence from III-V heterostructures during the tunneling process with nanometric resolution has been reported. ${ }^{9}$

The experiment presented in this article involves the inverse process. Surface plasmons optically excited on a silver film are shown to induce an additional current of electrons from the tip to the sample. This current is superimposed on the tunneling current observed in absence of surface plasmons.

The experimental setup allows one to investigate for the first time the spatial distribution of the ac-electric field induced by the plasmons with a resolution in the range of nanometers. This may help towards a better understanding of the processes leading to surface enhanced spectroscopy effects, where local field enhancement at specific sites at the surface (e.g., at points with large curvature, pores below the surface, or at sites of chemisorption) is believed to play an important role. ${ }^{10}$

\section{EXPERIMENT}

The experimental setup is sketched in Fig. 1. The STM used for the experiment has been described in detail elsewhere. ${ }^{11}$ The coarse approach is performed by piezodriven micrometer screws allowing a precise positioning of the tunneling tip relative to the sample in all three dimensions. Vibrational insulation is provided by a stack of metal plates separated by pieces of viton. The tunneling tips have been prepared by electrochemical etching of a tungsten wire of 0.5 $\mathrm{mm}$ diameter with $2 \mathrm{~mol} \ell^{-1} \mathrm{KOH}$.

The surface plasmons are optically excited in a Kretschmann configuration. ${ }^{12}$ The light of a $5 \mathrm{~mW}$ HeNe laser is scanneu at a frequency of $1.3 \mathrm{kHz}$ over a small angle by a mirror M1 (see Fig. 1) mounted on a bimorph piezo. A telescope optical arrangement (lenses L1 and L2), then images the angularly modulated beam onto a spot of about 0.5 $\mathrm{mm}$ diameter on the silver film, thermally evaporated onto the prism base. The mirror M2 allows for positioning of the spot. The attenuated total reflection signal is collected by a lens (L3) and detected with a photodiode.

\section{RESULTS}

Figure 2 shows synchronized traces of the signal on the photodiode (a) and the tunneling current for a tip voltage of

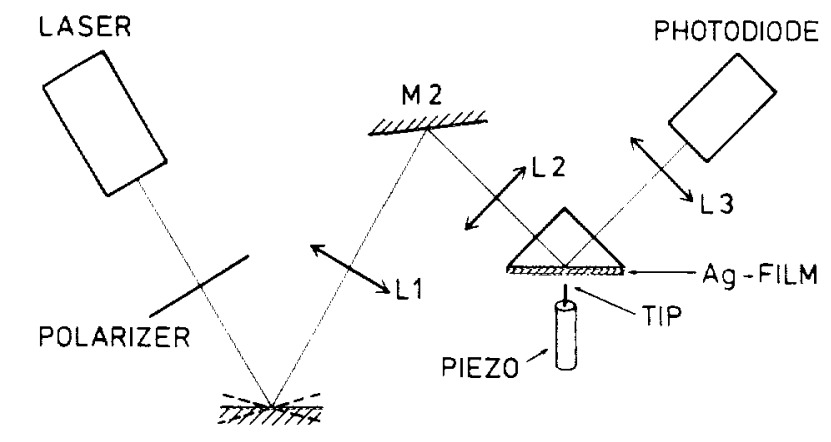

FIci. 1. Schematic representation of the experimental setup. 


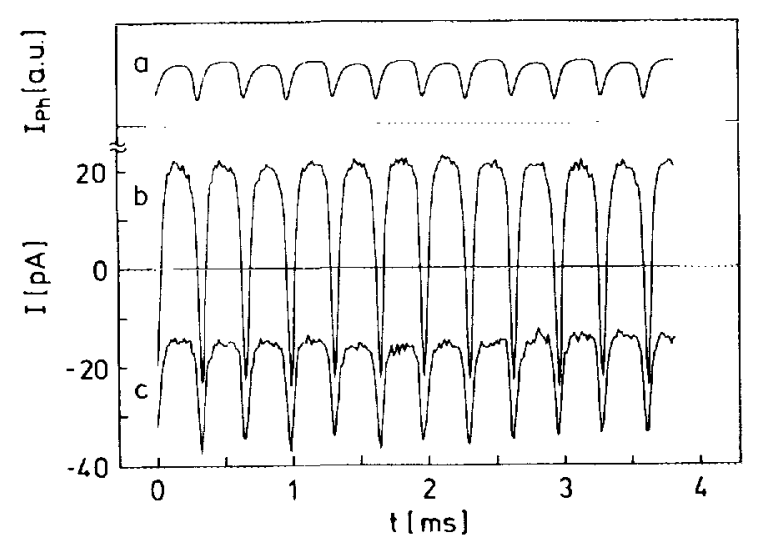

FIG. 2. Correlation between the excitation of surface plasmons and the tunneling current detected by STM: (a) signal of the photodiode showing the attenuation of the total reflection if surface plasmons are excited; (b) tunneling current at a tip bias of $+50 \mathrm{mV}$, the average value of the tunneling current is $20 \mathrm{pA}$ : (c) tunneling current at a tip bias of $-50 \mathrm{mV}$, the average value of the tunneling current is $-20 \mathrm{pA}$.

$+50 \mathrm{mV}(\mathrm{b})$ and $-50 \mathrm{mV}(\mathrm{c})$. The excitation of surface plasmons is characterized by the "dips" in the signal of the photodiode due to the attenuated total reflection. It can be clearly seen that a correlated effect is observed in the tunneling current. The absolute value of the average tunneling current has been set to $20 \mathrm{pA}$ for both curves (b) and (c). The positive sign of the current corresponds to electrons tunneling from the sample to the tip. For positive bias at the tip the tunneling current is reduced when the surface plasmons are excited.

For the particular parameters of trace (b) the current is even reversed. At negative tip bias the tunneling current is enhanced. The relative intensity of the plasmon induced signal (in the following named PS) is smaller, since for the given $I-V$ characteristic [Fig. 5 (b)] the gap distance is increased at negative tip bias if the absolute value of the tunneling current is maintained. On the observed time scale (the plasmon excitation lasts about $70 \mu \mathrm{s}$ ) the effect appears to be instantaneous.

Since the sign of the PS is independent of the bias voltage between tip and sample it can be excluded that the observed effect is caused by thermal expansion. The latter always leads to an increase of the absolute value of the tunneling current.

To get more insight into the nature of the coupling between the surface plasmons and the tunneling current the latter has been measured as function of the gap distance between the tunneling tip and sample surface. Figure 3 shows the PS (a) and the tunneling current (b) as function of the gap distance. While the tunneling current decreases exponentially the PS signal shows a complex behavior. It reveals only a weak distance dependence in the range of typical tunneling parameters. If the gap distance is further increased the decrease of the PS becomes stronger. However, even at the maximum gap distance in Fig. 3 where no tunneling current was detectable in the absence of surface plasmons the PS still amounts to about a third of the value at the typical operating conditions.

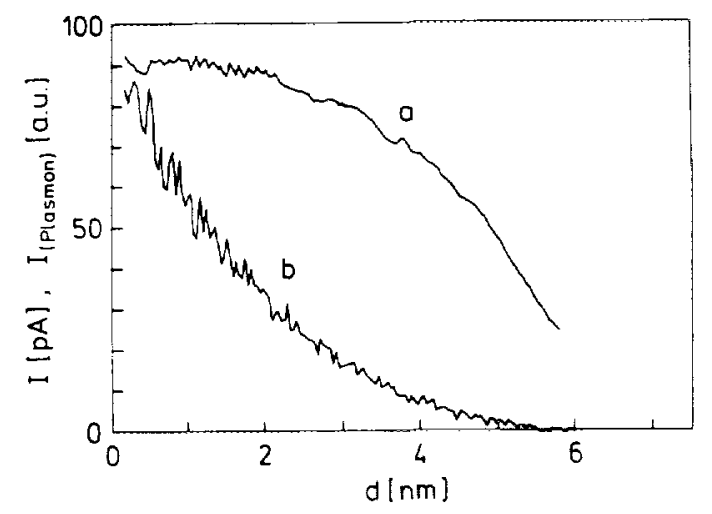

FIG. 3. PS and tunneling current as a function of the distance between tunneling tip and surface sample: (a) plasmon induced signal (PS): (b) tunneling current in the absence of plasmons.

This distance dependence leads to the conclusion that the observed effect is neither due to a surface photovoltage nor due to a thermovoltage caused by differential thermal heating. In both cases a simple exponential dependence is to be expected as the photovoltage or the thermovoltage act as constant voltage source in series with the applied bias.

The data displayed in Figs. 2 and 3 have been measured at a particular site on the sample showing a strong PS. To investigate its local intensity distribution the PS has been recorded simultaneously with the topography of the silver surface observed by STM. The upper part of Fig. 4 shows the topography of an area of about $60 \times 60 \mathrm{~nm}$ obtained at a tip bias of $150 \mathrm{mV}$ and a tunneling current of $20 \mathrm{pA}$. To measure the PS the output of the current-to-voltage converter amplifying the tunneling current is connected to a lock-in amplifier. The angle of incidence of the laser beam is varied sinusoidally. Since the plasmons are excited at the center of the scan range, the plasmon signal can be detected by using the second harmonic of the scan frequency as reference for the lock-

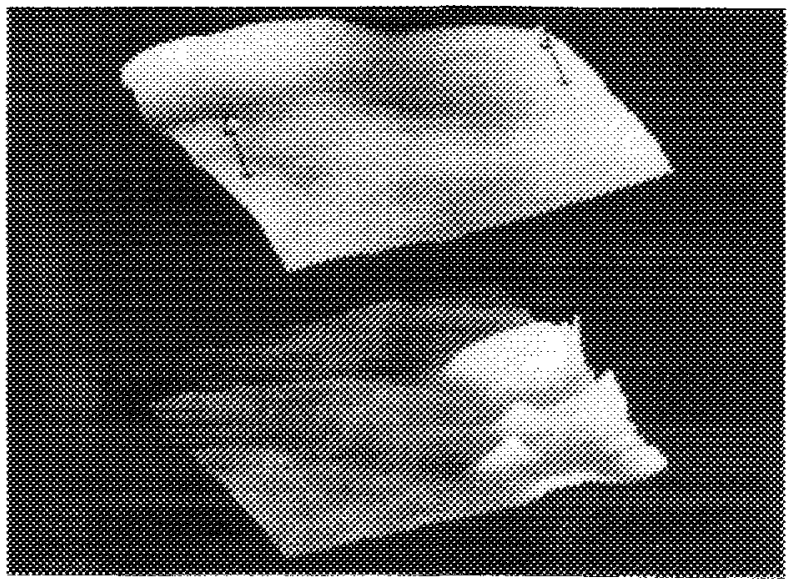

FIG. 4. Simultaneous recording of the surface topography and the plasmon induced signal (PS). The investigated area is about $60 \times 60 \mathrm{~nm}$, the tip bias is $150 \mathrm{mV}$, the tunneling current is $20 \mathrm{pA}$. Two positions at which the $I-V$ characteristics displayed in Fig. 5 have been measured are marked (a) and (b). The upper part shows the topography observed by STM; the lower part gives the corresponding plasmon induced signal 
in amplifier. The correlated PS is displayed in the lower part of Fig. 4. It can be seen that for a large part of the surface the signal is weak and only on particular areas a strong effect appears. The weak signals correlated with the topography but no particularity of the sites exhibiting a strong PS can be recognized in the topography.

To understand the local variation of the PS the tunneling current has been measured as function of the applied bias voltage for a site showing no PS (marked a in Fig. 4) and one with a strong PS (marked b). The resulting $I-V$ characteristics for $V$ varying from -150 to $+150 \mathrm{mV}$ are displayed in Fig. 5. At the position marked (a) a nearly linear dependence is found. A very different curve is found at (b) where strong PS is observed. The characteristic shows a strong nonlinearity; a strong increase of the absolute value of the tunneling current is found at negative tip bias. The surface plasmons are excited periodically, the dips in the curve represent the PS.

The nonlinear form of the $I$ - $V$ curve appears to be characteristic for the spots showing a strong PS. This behavior has been found whenever PS is observed. The nonlinearity of the $I$ - $V$ curve represents a necessary condition, a PS is, however, not always observed if a nonlinear $I-V$ curve is measured.

The experimental findings strongly suggest that the observed effect is due to the rectification of the ac field of the surface plasmon by the nonlinearity of the $I-V$ dependence of the tunneling gap. Although it is questionable whether the quasistatic $I-V$ characteristic measured at low frequencies is applicable as well as for optical frequencies of $5 \times 10^{14} \mathrm{~Hz}$, the experimental results agree well with the conclusion drawn from the static $I-V$ curve. Therefore the PS should increase with the "curvature" of the $I-V$ curve, i.e., if a strongly curved part of the $I-V$ curve is selected by choosing the corresponding bias. Measurements performed with the lock-in amplifier reveal that the PS increases by $50 \%$ if the bias at the tunneling tip is changed from +50 to $-50 \mathrm{mV}$ at constant gap distance.

We assume that the distance dependence of the PS is to a great part due to changes of the $I-V$ characteristic as a function of distance. This might also explain the complex distance dependence discussed above. For large gap distances

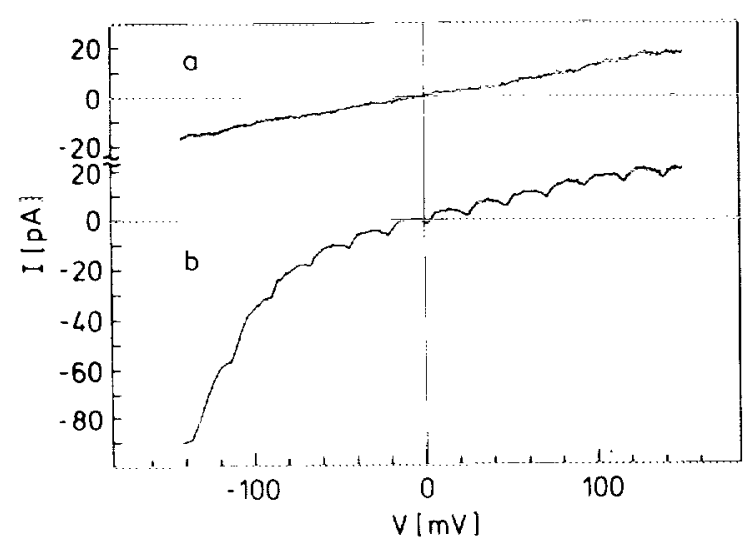

Fici. 5. Comparison between two different current-to-voltage curves: (a) linear $I-V$ curve measured at the position (a) in Fig. 4 where no strong PS has been detected; (b) nonlinear $I-V$ curve measured at the position (b) in Fig. 4 where a large PS has been detected. the relative increase of the curvature with growing gap distance then compensates for the overall decrease of the tunneling current.

\section{CONCLUSIONS}

The excitation of surface plasmons in a silver film leads to an additional flow of electrons from the tunneling tip to the silver surface. Since the PS is only observed if the $I-V$ characteristic shows a strong nonlinearity it is concluded that the observed signals are due to rectification of the AC field of the surface plasmon. Using the static $I-V$ curve one can estimate the $\mathrm{AC}$ voltage across the gap which is required to generate the observed signals. For a strong PS values of about $100 \mathrm{mV}$ are obtained. The effect of rectification of an ac field by the nonlinear $I-V$ curve of the tunnel junction has been discussed by Cutler $e t a l^{2}$ for the case that the laser light is shining directly onto the junction. The distance dependence of the PS found in our experiment shows a good agreement with their theoretical prediction.

However, our results do not confirm the theoretical prediction by Tagliacozzo and Tosatti ${ }^{13}$ that surface plasmons should lead to a lowering of the potential barrier for the tunneling electrons resulting in an increase of the tunneling current independent of the polarity of the applied bias voltage. If such an effect is present it has to be weak in comparison to the rectification discussed above.

The local distribution of the PS varies dramatically. It is assumed that the large signals are observed if the $I-V$ characteristic is strongly curved and if a strong local electric field is present. The experiments have been performed in air, hence the $I-V$ curve can be completely changed by impurities. However, it should be possible to prepare the sample and the tunneling tip under UHV conditions in a way that a nonlinear $I-V$ curve is obtained which shows only little local variation. Therefore one should be able to map directly the local electric field.

We believe that the coupling of laser light into the tunneling gap by means of surface plasmons opens up a new field of experiments. Since the laser intensity used in our experiment is several orders of magnitude smaller than in other experiments combing laser radiation and STM, thermal effects are negligible. The range of available wavelength covers the infrared and the visible spectrum. The experimental arrangement is therefore ideally suited to investigate, e.g., adsorbed atoms, molecules, etc., in the presence of an electric field of optical frequency by STM.

\section{ACKNOWLEDGMENTS}

We thank J. Y. Grand and H. Geoffray for their helpful assistance. Part of this work is supported by the Deutsche Forschungsgemeinschaft (SFB 306).

'N. M. Amer, A. Skumanich, and D. Ripple, Appl. Phys. Lett. 49, 137 (1986).

'P. H. Cutler, T. E. Feuchtwang, T. T. Tsong, H. Nguyen, and A. A. Lucas, Phys. Rev. B 35, 7774 (1987). 
${ }^{3}$ W. Krieger, T. Suzuki, M. Völcker, and H. Walther, Phys. Rev. B 41, 10229 (1990).

${ }^{4}$ L. Arnold, W. Krieger, and H. Walther, Appl. Phys. Lett. 51, 786 (1987).

${ }^{5}$ R. J. Hamers and K. Markert, Phys. Rev. Lett. 64, 1051 (1990).

${ }^{6}$ Y. Kuk, R. S. Becker, P. J. Silvermann, and G. P. Kochanski, Phys. Rev. Lett. 65, 456

'J. K. Gimzewski, J. Sass, R. R. Schlittler, and J. Schott, Europhys. Lett. 8, 435 (1989).

${ }^{8}$ R. Berndt, A. Baratoff, and J. K. Gimzewski, Proceedings of the NATO Meeting, Erice, Italy, April 17-29, 1989 (Kluwer Academic).
${ }^{9}$ D. L. Abraham, A. Veider, Ch. Schönenberger, H. P. Meier, D. J. Arent, and S. F. Alvarado, Appl. Phys. Lett. 56, 1564 (1990).

${ }^{2}$ See, e.g., H. Metiu, Prog. Surf. Sci. 17, 153 (1984); M. Moskovits, Rev. Mod. Phys. 57, 783 (1985); A. Otto et al., Surf. Sci. 210, 363 (1989).

" R. Möller, A. Esslinger, and M. Rauscher, J. Vac. Sci. Technol. A 8, 434 (1990).

${ }^{12}$ E. Kretschmann and H. Raether, Z. Naturforsch. A 23, 2135 (1968).

${ }^{13} \mathrm{~A}$. Tagliacozzo and E. Tosatti, "Tunneling with coupling to a surface phonon or plasmon," in Proceedings of the Adriatico Research Conference on "Scanning Tunneling Microscopy," July 28-31, 1987. 